\title{
COMPUTER SIMULATION OF TWO-PHASE FLOW IN NUCLEAR REACTORS
}

\author{
Wolfgang Wulff \\ Brookhaven National Laboratory* \& BNL-47783 \\ DE92 040565

\begin{abstract}
Two-phase flow models dominate the economic resource requirements for development and ise of computer codes for analyzing thermohydraulic transients in nuclear power planis. Six priaciples are presented on mathematical modeling and selection of numerical methods, along with suggestions on progiamning and machine selection, wll aimod at reducing the cost of analysis.

Computer simulation is contrasted with traditional compuler calculation. The advantages of run-time interactive access operation in a simulation environment are demonstrated.

It is explained that the drift-flux model is better suited for two-phase flow analysis in nuslear reactors than the two-fluid model, because of the latter's closure problems. The advantage of analytical over numerical integration is demonstrated. Modeling and programming tochniques are presented which minimize the number of ateded arithmetical and logical operations and thereby increase the sinulation speed, while decreasing the cost.
\end{abstract}

\section{INTRODUCTION}

The cost of computing, that is of executing arithmetical and logical operations on an electronic digital computer, has dramatically declined in recent years, but the total cost of analyzing transient two-phase flow phenumena in, and the dynamic behavior of, complicated nuclear reactor systems has not significantily decreased. The cost of initial cupital investment, maintenance and operation of newly available workstations are small compared to those for the equivalent computing capucity in previously used supercomputers. The overall cost of analyzing complex systems has not decreased, because it is dominated by the analyst's salary', which in tura is dictated by the time it takes to prepare, execute, unalyze and document the several computer calculations normally neaded to assess a single reactor transient and the uncertainty associated with the analysis.

Efforts of analyzing normal and accident transients in nuclear reactor power plants are dominated by the difficulty to rnodel and numerically simulate a vast nut.aber of multiphase flow phenomena in the reactor coolant and possibly in the core melt. Multiphase, and particularly two-phase flow issues will therefcre be the focus of this paper.

There are three fundamental reasons for the widely experienced, painfully large expenses of nuclear reactor unalyses: (i) the demand for growth of scope and detail of the analyses could not be compensated for by advances in computing speed, (ii) the models and computer codes currently used for reactor analyses are largely inefficient, and (iii) the analysis methods are oriented toward computer calculation, rather than toward faster, rnore expeditious, convenient and efficient computer simulation.

Reason (i) arises from the need to account for an increasing number of more and more complicated phenourena in the analysis. This need will remain and must be dealt with, as reactor technologies advance and

This work was performed under the auspices of the U. S. Department of Energy. 
2

as the objectives expend from design base to severe accident analyses. The expectation, bowever, of solving the problems of high cost and long exarution time for computer analyses, by waiting for low-cost computers of "ultima'e" speed, is not realistic on the basis in history.

Rensons (ii) and (iii) are the subject of this puper. It will be demonstrated that engineering judgement in selecting and formulating models, in selecting established and documented numerical methods, and that working in what will be described as a simulation enviroument, can significantly improve the quality of twophase flow analyses, while reducing the time and cost that they require.

BWR stability analyses performed recently by Wulff et al $[1,2,3]$, in the simulation environment of the BNL Engineering Plant Analyzer [4] on the one hand, and with INFI's standard FORTRAN code TRAC. BF1 [5], nun on the CRAY general-purpose supercomputer, had the sume objectives: to analyze (with point kinetics) core wide oscillations related to those that had occurred at the LeSalle-2 Power Station in March of 1989. Approximately the same timo and expenditures provided from the vinulation environment over ninety different, complete plant transient analyses, including all systems feedback effects [1], and from the standard FORTRAN coinputins method the effects of four parameter variations on open loop (reactor core channel) calculations with imposed boundary conditions. By working in a simulation, rather than in a computing environment, and by appropriate modeling techniques, one could reduce the total effort of analyzing reactor transients by about two orders of magnitude.

The objectives of this paper are to explain the difference between computing and simulation, to present six principles of modeling and numerical methods, all of which are aimed at reducing ihe computational effort. It is also the objective of this paper to suggest three coding requirements and to present the important features of the simulation computer environment. Lastly, it will be shown why modeling and numerical methods need to be optimized with the computer architecture as a whole. A given computer code, when optimized for a given computer, cannot achieve the same degree of optimization on another computer, primarily when intensive input and output processing is involved.

\section{COMPUTING VERSUS SIMULATION}

Traditional computer analyses of nuclear reactor transients, involving two-phase tlow and proceeding both under normal and postulated accident conditions, have been performed by computer calculation. Computer calculations are carried out on generil purpose computers (super, mainframe, minicomputers or workstations), using an operating system, a FORTRAN compiler and a standard FORTRAN computer code, such as RETRAN $[6,7,8]$, RELAP5 $[9,10,11]$ or TRAC $[12,13]$. The codes are executed in batch mode, and the results are postprocessed with a gruphics code to display them conveniently.

In contrast, computer simulation is carried out in a simulation environment, consisting, not necessarily but preferably, of a spocial-purpose simulation computer, and consisting always of an interactive simulation software system, a sirnulation language and a simulation code which describes the physics of the system to be analyzed. The interactive simulation software system is the most distinctive characteristic of the simulation environment, followed by the input and output processing hardware components of the simulation computer, and then by the simulation language.

Computer simulation is carried out in-line, interactively. Herein lies the main reason for its efficiency. It provides the analyst with run-time control for setting parameters, activating simulated component functions o. failures, resetting initial conditions, further for reselecting integration algorithms and display fearures. All of this is achieved without relording and recompiling the program. The analyst can participate in the simulation, by monitoring and influencing the same, in-line, during its execution. This is very important for the assessment of operator actions and for carrying out efficiently large numbers of scoping calculations. 
Hardware, such as analog or digital sensors and control systems, can be tested and optimized in the computational loop of a simulation facility. Any program variable can be displayed versus any other variable or time, without preprogramming or reloading the program, and in uny form, such as the trend on an oscilloscope, or in tabular form. Herein lies the simulation environment's strength for code development.

The simulation language, such as EASYS [14], ACSL [15] or ADSIM [16], is state variable oriented and has a large number of simulation-specific constructs, including a choice of proven and documented methods for numerical integration and matrix algebra, and for multidimensional table generation and interpolation.

Computer simulation is today most efficiently achieved with special purpose computers, such as the AD-100 [17] in the BNL Engineering Plant Analyzer. Still, no alternative can reach its convenience and computing speed, primarily bocause of its superior input and output data processing. However, todsy's special-. purpose simulation computer capabilities can be achieved on advanced workstations, once their simulation systems software is developed and the high-speed, high capacity input processing capabilities (hardware and software) are available.

\section{SIMULATION PRINCIPLES}

The four elements of simulation are the mathernatical models which define the physical system to be simulated, the numerical methods with which to integrate and solve the equations of the mathematical model, the simulation language which facilitates the execution of the numerical methods on a computer, and an interactive computing facility.

\subsection{Modeling Principles}

Three modeling principles are presented here to promote modeling efficiency, which is achieved when the greatest possible simulation fidelity is obtained with the smallest possible number of arithmetical and logical operations.

\subsubsection{Principle of Model Selection}

Model seluction for gas-liquid two-phase flow simulation is important, because a variety of models with differing modeling idetail and complexity is available, and the choice one takes has a profound impact on modeling fidelity and simulation cost. Two-phase flow models are categorized by the number of conservation equations employed for mass, momentum and energy conservation in the liquid and the gas, and the related numbers of implied constraints and needed transfer laws for closure.

Wulff [18] related in 1981 systematically the number of conservation equations to the numbers of constraining assumptions and transfer laws needed to relax constraints. He covered the range from the simplest three-equation model for honsogeneous equilibrium two-phase flow, which carries the maximum of three limiting constraints of equal pressures, equal temperatures and equal velocities for both phases, to the most complex, six-equation two-fluid model for nonhomogeneous, nonequilibrium two-phase flow, which has no constraints.

The three equation model requires two transfer laws, one each for wall shear and wall heut transfer. The six-equation model requires seven laws, three for mass, momentum and energy iransfer between the pheses, and two each for momentum and energy iransfer between the wall and each phase. In general, multiphase flows with a phases, modeled by $3 n$ conservation equations, require $n(3 n+1) / 2$ flow patterndependent transfer laws. 
Complete closure requires additional constitutive laws for material descriptions and interface conditions. Isbii established in 1975 [19], that the two-fluid model formulation contains thirty-two variables and sequires twew.y-six closure relstions.

The principle of model selection is that one should choose the least complicated model which accommodates all available experimental information and the phenomena of interest.

Irrespective of its complexity, no model can produce more information than is contained in its data base, which includes the closure laws. On the other band, intolerable simplifying assumptions (such as bomogeneous vertical flow) render a model inadequate.

Modeling complexity is linked to computational complexity and corresponding efforts of computing. Madels which cannot produce information commensurato with their complexity squander computing resources.

The application of the principle of model selection to two-phase flow modeling leads to the conclusion that the two-fluid model can be justified at this time only for separated flows, where the interface geometry is known and amenable to boundary layer analyses. Transfer laws for momentum exchange between phases and a review of the currently inadequate capability of predicting interfacial area density for the two-fluid model were published by Ishii [20]. The two fluid model is also suitable for testing modeling hypotheses, and for linking theory with experiments.

For the chaotically deforming interfaces of two-phase flow patterns occurring in nuclear reactors, however, there is still no experimental basis for supporting the two-fluid model by developing transfer laws to describe the split of total inso phasic momentum and energy transfers at the wall; there is still no suitable instrumentation available for developing such transfer laws.

The four-equation drift flux model introduced by Zuber and Findlay [21] in 1965 is a mixture model, akin to the familiar multispecies model of chemical engineering. It accommodates directly better than any other two-phase flow model the available experimental information. Relative phasic velocities are predicted in terms of two flow-regime dependeat parameters: the drift velocity and the distribution parameter, as published by Ishii [22]. According to Boure [23], the drift-flux model is especially well suited for predicting the propagation of thermal and kinematic disturbances, which are precisely the objectives of thermohydraulics simulations. The comparison of the performances of the drift-flux model and the two-fluid model, as documented in references [1] through [4] on the ocie hand and in [5] on the other, supports this conclusion.

The closure problem for the drift-flux model is smaller: there is no wall shear and wall heat flux splitting required. The integration of four equations requires obviously fewer resources than that of six equations. The drift-flute model is superior to the two-fluid model for the prediction of flows with tightly coupled phoses and stochastically defornning interfaces.

\subsubsection{Scaling and Model Simplification}

The second modeling principle calls for an order of magnitude comparison, to be performed on all the terms of the governine mojtel equations and, based on it. for the elimination of all terms which represent irrelevant phenomens.

This model simplification is subject to the requirement that well-posedness must be preserved: the elimination of seemingly unimportant dissipative terms must not change a well-posed parabolic into an ill-posed hyperbolic system of partial differential equations having complex characteristics. 
Typicat simplifications are the elimination of virtual mass effects, except where large local accelerations are to be modeled, and the complete elimination of acoustic effects from thermohydraulic codes (by setting $p p=0$ in mass and energy balances), unless these are to predict dynamic interactions between fluid and structure.

The suggested simpitinication process is similar to the normalization of the governing equations, normally performed for scaling and the purpose of compacting the large number of constants in the equations into a much smaller number of scaling groups. Just as only two scaling groups, the normalized wall roughness and the Reynolds number suffice completely to describe wall shear in all circular tubes and for all liquids and gases, so also facilitate scaling groups a significant reduction in the number of needed arithmetical operations. Thus, scaling of equations reduces the computational effort, even without the simplification of equations.

Finally, scaling in computer analyses would focus attention on the need to cover the range of full-scale reactor plant conditions both by smiall-scale experiments and by selecting correlations for the models.

\subsubsection{Use of Analytical Methods}

The third modeling principle urges that analvtical rather than numerical integration be used. whenever that is feasible, and that analytical solutions be dynamically evaluated on the computer for the conditions evolvine durine a transient.

Analytical solutions are easy to assess regarding the effects of, and uncertainties from, simplifying assumptions, all of which are enumerable. Analytical solutions are free from effects of numerical diffusion and raise no concerns about convergence.

The analytical integrations over space of the mixture momenturn and mass balances are explained below to demonstrate the benefits from analytical methods in two-phase flow simulation.

The analytical integration of the mixture momenturn equation around every closed loop in the system is based on Meyer's momentum integral model [24] and has been used in the BNL Engineering Plant Analyzur [4] with great success to reduce over fifty partial difference to just three ordinary differential equations, one for every closed loop in the system. The loop momentum is defined as

$$
M=\oint \frac{W_{m} d z}{A} \text {, }
$$

and computed from the loop integral of the momentum balarce:

$$
\begin{aligned}
\frac{d M}{d t}= & \Delta p_{p}-\sum_{k=1}^{N}\left(\Delta \Psi_{m}\right)_{k}-\oint g_{z} \rho_{m} d z-\sum_{k=1}^{N}\left(L\left\langle f_{z}\right\rangle\right)_{k} \\
& -\sum_{j=1}^{N+1}\left(\Delta p_{D}\right)_{j}+\left(\Psi_{m}\right)_{i n j} \frac{A_{i n j}}{A} \cos \theta .
\end{aligned}
$$

In Eq. 1, $A$ and $W_{m}$ stand for the flow cross-sectional area and mixture mass flow rate, respectively, while $z$ denotes the courdinate axis along the direction of flow. In Eq.2, the first term on the right is the pressure nue across the pumps. The second term is the change of mixture momentum flux $\Psi_{m}=w_{1} G_{1}+w_{v} G_{v}$ in a lixpp component of constant cross-section; here $w$ and $G$ stand for velocity and mass fiux, respectively, subscripts

$I$ and $v$ denote liquid and vapor. The third term represents gravity, the fourth term mixture wall shear, the lith term form losses, and the last term the momentum change due to injection. 
It can be seen that there are no restrictions introduced by deriving Eq.2. The evaluation of Eq.2 implies, however, that phasic densities are computed with the local enthalpy and volume average pressure; the insignificant dependence on local pressure variation is ignored. Therefore, pressure wave propagation cannot be predicted; pressure signals are modeled to propagate instantly around the loop.

There are four advantages of integrating Eq. 2 instead of the original partial differential equation: (i) As any closed contour integral of the pressure gradient is zero, Eq.2 has no pressure term; (ii) Equation 2 reduces a partial to an ordinary differential equation and quadratures over space, therefore, it reduces the computational effort of integrating the momentum balance; (iii) the numerical stiffness of Eq.2, dominatud by loop length over flow cross-sectional area, is sharply reduced relative to that of the corresponding set of finite difference equations, high-frequency ringing is eliminated, larger integration time step sizes are permitted, and (iv) Eq. 2 introduces no numerical dissipation from discretizing the momentum flux term.

Equation 2 is needed to model, with the help of the mixture mass balance and drift velocity correlations, the time-dependent phasic velocity variations throughout the two-phase flow system. Equation 2 produces a loop momentum $M$ for every closed loop of a system, each $M$ depending linearly on the volumetric mixture flow rate $\left(\beta_{\mathrm{m}}\right)_{0}$ at a conveniently chosen loop location $\mathrm{z}=0$. A set of $\mathrm{n}$ momenta yields therefore one volumetric mixture flow rate for each of $\mathbf{n}$ loops, and this flow rate $\left(\beta_{m}\right)_{0}$ serves as the needed integration constant for the spatial integration of the mixture mass balance, fornulated as the mixture flux divergence equation.

This is easy to see, because in the drift-flux formulation, the mass flow rate $W_{m}$ in Eq.1 depends on the volumetric flow rates $\beta_{m}$ ot mixture and vapor $\beta_{v}$, which are expressed in terms of the phasic density difference $\Delta \rho=\rho_{1}-\rho_{v}$, the vapor void fraction $\alpha$, the distribution parameter $C_{0}$ and the void weighted, area averaged drift velocity $\left\langle v_{\mathrm{gj}}\right\rangle$ as follows:

$$
\begin{aligned}
W_{m} & =\rho_{1} \beta_{m}-\Delta \rho \beta_{v} \\
& =\left(\rho_{1}-\alpha C_{0} \Delta \rho\right) \beta_{m}-\alpha \Delta \rho<v_{g j}>A
\end{aligned}
$$

and $\beta_{m}(z, t)$ is known from the mixture mass balance only up to the integration constant $\beta_{m}(0, t)=\left(\beta_{m}\right)_{0}$.

This leads to the analytical integration of the mixture mass balance, the second example for demonstrating the advantages of analytical over numerical methods in two-phase flow modeling. After defining the mixture and phasic fluxes $j_{m}=j_{1}+j_{v}=(1-\alpha) v_{1}+\alpha v_{v}$, the two-phase mixture mass balance is written as the mixture volumetric flux divergence equation

$$
\nabla \cdot J_{m}=\frac{\rho_{1}-\rho_{v}}{\rho_{1} \rho_{v}} \Gamma_{v}-\frac{\alpha}{\rho_{v}} \frac{D_{v} \rho_{v}}{D t}-\frac{1-\alpha}{\rho_{1}}-\frac{D_{l} \rho_{1}}{D t}
$$

where $r_{v}$ is the vapor generation rate and $D_{k} / D t$ is the su istantial derivative, computed with the k-phase velocity.

The substantial derivatives are evaluated with the thermal equations of state, in terms of pressure and energy derivatives, and the latter are eliminated with the phasic energy equations. The result is first integrated over the system volume to yield the rate of pressure change. For the vapor at saturation (at low vapor void fractions), one finds, with the Gauss divergence theorem, the time rate of pressure change 


$$
\frac{d p_{\text {sys }}}{d t}=\frac{\iiint\left(F_{\Gamma} \Gamma_{V}-F_{q}(1-\alpha) q_{L}^{\prime \prime \prime}\right) d V-\sum_{\text {outflow }} \beta_{m}+\sum_{\text {comp. }} F_{q} \oint_{\vec{q}_{\dot{w}}} d \vec{A}}{\iiint F_{p} d V}
$$

where

$$
\begin{aligned}
& F_{\mathrm{r}}=\frac{\rho_{1}-\rho_{V}}{\rho_{1} \rho_{V}}+F_{q}\left(h_{g}-h_{l}\right) \\
& F_{p}=\frac{\alpha}{\rho_{g}} \frac{d \rho_{g}}{d p_{p}}+\frac{1-\alpha}{\rho_{1}}\left(\frac{\partial \rho_{1}}{\partial p}\right)_{u} \\
& F_{q}=\frac{1}{\rho_{1}^{2}}\left(\frac{\partial \rho_{1}}{\partial u_{1}}\right)_{p}
\end{aligned}
$$

The numerator of Eq.5 contains the dilation effocts from phase change and gamma heating, from net-discharges and from wall heating, in thet order, while the denominator contains the system compressibility.

The previous result is integrated a second time, now over any portion of the system volume, such that the enclosing surfaces are crossed only by known and one unknown volume flow rates $\beta_{m}=A j_{m}$, to give

$$
\begin{gathered}
\beta_{m}(z, t)=\left(\beta_{m}\right)_{0}(t)-\frac{d p_{s y s}}{d t} \iiint F_{p} d V+\iiint F_{\Gamma} \Gamma_{V} d V-\sum F_{q} q_{w}^{\prime \prime} A_{W} \\
-\iiint F_{q}(1-\alpha) q_{I}^{\prime \prime \prime} d V
\end{gathered}
$$

Equation 7 constitutes the integral of the mixture mass balance and predicts the mixture volumetric flow rate at any location in the loop, in terms of the loop integration constants $\left(\beta_{m}\right)_{0}$, which are defined by the loop mixture momentum balances, and the mixture mass balances for suitable branch points. The branch points for PWR simulation are the connection of the hot legs with the upper plenum, and the core entrance; for BWR simulation, the branch point is the core entrance.

The advantages of Eq.7 over a finite difference equation are: (i) it reduces a partial to an ordinary differential equation and quadratures over space, therefore, it reduces the computational effort of integrating the mixture mass balance, and (ii) it introduces no numerical dissipation from disiretizing the density and velocity gradients in the mass balance. An even greater advantage arises from Eq.7 due to the choice of volumetric, rather than mass flow rates, because the volumetric flow rate $\beta_{\mathrm{m}}$ is continuous under most circumstances across discontinuities of void fraction, such as a liquid or a mixture level.

The phasic volumetric flow rates are computed everywhere and for both flow directions from the above mixture volumetric flow rates and drift-flux correlations. To accommodate flow reversal at branch points, one needs to find the individual vapor flow rates. If the flows are taken positive in the trunk (subscript 1) toward the plane of the branch and in the branches (subscript $k$ ) away from the plane, then one geis from the vapor and mixture mass balances, for perfect lateral mixing in, and no phasic density change across, the plane of branching 


$$
\begin{aligned}
& \left(\beta_{v}\right)_{1}=1 / 2 A_{1}\left[\left(j_{v}\right)_{i}^{+}+\left|\left(j_{v}\right)_{i}^{+}\right|+\alpha_{i}\left[\left(j_{m}\right)_{1}-\left|\left(j_{m}\right)_{1}\right|\right]\right] \\
& \left(\beta_{v}\right)_{k}=1 / 2 A_{k}\left[\alpha_{i}\left[\left(j_{m}\right)_{k}+\left|\left(j_{m}\right)_{k}\right|\right]+\left(j_{v}\right)_{k}^{+}-\left|\left\langle j_{v}\right)_{k}^{+}\right|\right],
\end{aligned}
$$

where the superscripted plus sign denotes flow rates evalıated with fluid properties extrapolated from the channel interior, in the direction of flow, to just before the plane of branching. The void fraction at the plane is

$$
\alpha_{i}=\frac{\left(\beta_{v}\right)_{t o t}^{i}}{\left(\beta_{m}\right)_{\text {tot }}^{i}}
$$

where the net incoming vapor and mixture volumetric flow rates arriving at the plane are

$$
\begin{aligned}
& \left(\beta_{v}\right)_{\text {tot }}^{i}=1 / 2\left[A_{1}\left[\left(j_{v}\right)_{1}^{+}+\left|\left(j_{v}\right)_{1}^{+}\right|\right]-\sum_{\text {aldbranches }}\left[A_{k}\left[\left(j_{v}\right)_{k}^{+}-\left|\left(j_{v}\right)_{k}\right|\right]\right]\right] \\
& \left(\beta_{m}\right)_{t o t}^{i}=1 / 2\left[A_{1}\left[\left(j_{m}\right)_{1}+\left|\left(j_{m}\right)_{1}\right|\right]-\sum_{\text {allbranches }}\left[A_{k}\left[\left(j_{m}\right)_{k}\left|\left(j_{m}\right)_{k}\right|\right]\right]\right] .
\end{aligned}
$$

Equations 7 through 10 demonstrate that the flux divergence equation is a powerfull basis for simulating two-phase flow in multichannel, multiloop systems with flow reversals. It is also shown that analytical integration can reduce significantly the task of integrating partial differential equations of two-phase flow models. Analytical integration works equally well for heat transfer in structures and fuel $[24,25]$ and balance of plant components [4].

\subsection{Selection of Numerical Methods}

Computing efficiency is achieved when the best possible approximation to the solution of the modeling equations is found with the smallest possible number of arithmetical and logical operations, because that produces answers in the shortest possible time. The following three principles of selecting and implementing numericai metheds were shown to improve computing efficiency.

\subsubsection{Elimination of Iterations}

The first numerical methods principle calls for the elimination of iterative procedures, by pretabulating the results of iterations in terms of state, or subsidiary variables, which are known when the iesults are needed in a compurational cycle.

State variables form the minimum set of variables which define completely the state oi the simuluted system; they appear as the key time derivatives in the conservation equations and are always known at the beginning of a computational cj cle, when a state equation oriented simulation computer language is used. Subsidiary variables depend on state variables through algebraic or transcendental equations.

Coupled, nonlinear systems of equations occur always. An example is the dependence of wall temperature $T_{\text {wall }}$ on the convective heat transfer coefficient or Biot number $N_{g !}$, which depends also on the wall temperature. 


$$
T_{\text {wa1l }}-T_{f l u i d}=\frac{\left(\langle T\rangle-T_{t l u i d}\right)}{\left[1+N_{B i}\left(C_{g}+F_{D I}\right)\right]}
$$

The symbols $T_{\text {fluid }}\langle T\rangle, C_{g}$ and $F_{p r}$ in Eq.11 denote, respectively, the coolant temperature, the volumeaveraged strutural temperature, a geometric constant and a property function [4, p.3-29]. The dependence of $T_{\text {wall }}$ in Eq.11 is complicated, because branching is needed for flow regimes and because of complex correlations. The wall temperature can be found only by iteration.

Through the first numeric i methods principle, one utilizes now available, inexpensive computer memory; one replaces during the simulation large numbers of time consuming arithmetical and logical operations by a linear table interpolation on a multidimensional hypersurface, and one obtains directly the needed resul :.

In the above example, one would tabulate the needed wall heat flux directly in terms of coolant and structural temperatures (which are given through energy balances), of flow rate and void fraction (given through momentum and vapor mass balances). There is no need during the simulation for computing Reynolds, Prandtl, Nusselt and Biot numbers, nor for branching into the appropriate heat transfer regime, nor for computing heat transfer coefficients, nor for continuing to repeat that computation until the boundary condition of surface heat flux continuity in Eq.11 is satisfied.

\subsubsection{Use of Pretabulations}

The second aumerical methods principle calls for the pretabulation of complex expressions as multidimensional functions in terms of state, or subsidiary variables, which are known when the results are needed in a computational cycle.

While all parameters combining geometric constants are routinely precomputed as constani coefficients, possibly embedded in scaling groups, and while thermophysical properties had been pretabulated in the past, this numerical methods principle goes further. It serves to reduce, in principle, the computation of each element in the coefficient matrices $A_{i j}, B_{i j k}$ and $C_{i}$,

$$
A_{i j} \frac{\partial y_{j}}{\partial t}=E_{i j k} \frac{\partial y_{j}}{\partial x_{k}}+C_{i}
$$

of the governing conservation equations to a linear, multidimensional table interpolation. In praxis, however, the principle leads more likely to the pretabulation of complicated expressions, containing a combination of thermophysical properties and their derivatives [4, pp.3-100 and 3-105].

Pretabulation saves computing resources when (i) table interpolation takes less computing time $17 n$ the needed arithmetical and logical operations and (ii) the needed number of table entries can be accommodate with the available mennory capacity. Up to three function arguments present no memory problems in currently available minicomputers. The execution time of table interpolation for a function of a fixed number of arguments (table entries) is independent of the function complexity.

\subsubsection{Integration Methods}

The experience gained over the last decade has shown that there is a compelling advantage in employing well established and well documented algorithms for integrating the partial or ordinary (in time) 
differential equations of two-phase flow. One should use algorithms for which capabilities, limitations, discretization errors and convergence criteria are documented and can be used to control the computational error.

\subsubsection{Partial versus Ordinary Differential Equations}

The governing conservation equations for two-phase flow are modeled in lumper or discrete parameter form, and formulated either as ordinary or partial differential equations, respectively. As ordinary differential equations they are differential equations with respect to time and can be integrated by standard methods. Partial differential equations can be discretized in space and integrated over time as ordinary differential equations, or they can be completely discretized and integrated by solving a system of implicitly coupled nonlinear equations.

The simultaneous solution of large systems of coupled nonlinear equations requires iterations, is therefore time consuming and not recommended for simulation, except for very slow system response and steady state simulations. A precise recommendation for addressing system response frequency from the computational point of view is given next.

\subsubsection{Explicit versus Implicit Integration}

The choice between implicit and explicit numerical integration has a profound effect on computing efficiency. One must select that integration method which achieves the larger speed ratio $S=\Delta t_{i s t} I \Delta t$ frm, of permissible integration step size $\Delta t_{\text {ist }}$ over frame time $\Delta t_{f r m}$. Here, the integration time step is

$$
\Delta t_{1 s t}=\operatorname{Min}\left(\Delta t_{\text {acc }}, \Delta t_{s t b}, \Delta t_{s y s}\right) \text {, }
$$

where $\Delta t_{\text {acc }}$ is the maximum time step permitted by discretization errors, $\Delta t_{\text {stb }}$ is the maximum step size permitted by stability constraints, and $\Delta t_{\text {sys }}$ is the characteristic time (fraction of period) of the relevant system response. Relevant system response time in two-phase flow is the period of thermohydraulic oscillation, not of driving neutron kinetics transients.

The frame time is the time it takes the computer to complete a computational cycle, that is to advance from one time level to the next, by $\Delta t_{i g g}$. The permissible integration step size $\Delta t_{\text {iat }}$ increases with decreasing system response frequency, as the discretization error is proportional to the leading time derivative; for explicit integration it increases only up to the stability limit. Since implicit integration is unconditionally stable, $\Delta t_{\text {stb }}$ is infinite for implicit integration. However, the accuracy restricted $\Delta t_{\text {ncc }}$ is always larger for explicit than for implicit integration. It has been shown by Wulff [26], that for the same time and space discretization with donor-cell differencing, implicit integration has for all choices of time and space intervals always the greater numerical dissipation. Explicit integration methods for ordinary differential equations afford higher-order accuracy (by fourth-order Runge-Kutta method, for example) than first-order implicit solution schemes for the integration of space discretized partial differential equations of two-phase flow.

The frame time $\Delta \mathrm{f}_{\mathrm{fm}}$ is independent of the system response time as it depends only on the number of programmed operations and the computer's execution speed. The frame time $\Delta t_{f i m}$ is always much smaller fo: the direct computations of explicit, than for the iterative computations of implicit integration, the more so the larger the number of governing equations is.

For most transients (Large and Small Break Loss of Coolant Accidents, for example), one finds that $\Delta t_{a c c} \leq \Delta t_{s t b}$, and that explicis is superior to implicit numerical integration. Only for near steady-state conditions can $\Delta t_{\text {ace }}$ exceed $\Delta t_{\text {stb }}$ well enough to compensate for its frame time disadvantage and render the implicit better than the explicit method [26]. 


\subsection{Programming Principles}

Programming principles advance and support the development of simulation capabilities and, even more their applications.

\subsubsection{Quality Assurance}

Quality Assurance (QA) procedures have been developed for the nuciear industry, to document and audit code preparations, completion, checking, the control of cnde modifications and applications [27]. Aside from criteria of compliance between mathematical model formulation and coding, and from criteria of input data validation, the quality standards are subjective. The following two supplementary coding principles have been found to expedite code development and simulation.

\subsubsection{Simulation Languages}

H:gh-level simulation languages for computer programming have been recognized as superior to standard FORTRAN, when the application objective is simulation. Most simulation languages, such as EASY5 [14], ACSL [15] and ADSIM [16] compile on general-purpose computers through automatic translation into FORTRAN and are therefore transportable; ADSIM is at this time restricted to VAX computers and advanced workstations, but executes best on the special-purpose simulation computer AD-100 [16], which provides the integrated simulation environment described above in Chapter 2.

Simulation languages are specifically designed to minimize the task of translating mathematical models for physical systems into a computer program. They are state equation-oriented and have simulation-specific language constructs which facilitate code development, code validation, code documentation. A simulation program written in simulation language can be completely self-documenting and may be only half as long as the corresponding well written FORTRAN program. The vapor mass balance, for example, written in ADSIM, reads

$$
M v_{j} j^{\prime}=W v_{j}-1-W v_{j} j+v_{j} * \text { GAMMAv } j
$$

and resembles the modeler's notes. Each term could stand for a vector. There is no need to program, test and document an algorithm for integrating the above differential equation, as the superscripted prime automatically invokes, either a default or a deliberately selected, built-in integration procedure.

Simulation languages have the modular structure to assemble model blocks, function blocks and program regions with systematically identified interfaces. Simulation languages provide the capability to vary automatically key parameters and generate an output envelope by recycling through a set of simulations.

Simulation languages provide interactive run-time access for resetting system and program controls without interrupting the simulation. For these reasons, the use of simulation languages takes fewer economic resources to develop simulation code and to simulate, than the use of standard scientific programming languages.

\subsubsection{Self-Documenting Codes}

Simulation code should be self-documenting, or cite by page and equation numbers directly the model and its numerical implementation in the code documentation. All input parameters should be prompted on the monitor screen with engineering descriptions, including units, and stored automatically in the same record for auditing simulation and hard copy documentation. All output displays, graphical or tabular should be selfexplanatory. 
The preparation of input data, and the interpretation of tabulated output data still require for mose large systems codes extensive, time and resource consuming consultations of a user's manual. Small efforts for improving code commenting and input and output labelling could improve the efficiency of code use.

\subsection{Simulation Computer Architecture}

Simulation computers have simulation task-specific processors, working in parallel to provide run-time interactive access, to carry out operations of arithmetic and logic, multidimensional table interpolation, integration, etc. These processors bave their own built-in instructions (firm ware) and they interact with minimum overhead, in lockstep and through broadcasting over a high-speed bus.

The most distinguishing feature of simulation computers is their separate and dedicated communications bus which, in conjunction with an input-output processor, affords high-speed digital and analog signal transmissions to and from peripheral devices. This hardware and the associated special input-output software, along with the software for run-time interactive access make the simulation computer superior to high-speed general-purpose computers, including workstations, because its input and output processing does not reduce the simulation speed. Moreover, a simulation computer provides ror simulation with, and testing of, hardware in the loop.

\section{SYNERGY OF SIMULATION ELEMENTS}

Experience has shown that efforts to vectorize an existing code, or to add on post-processing for graphic displays, generate only modest improvements in efficiency. The most efficient simulation performance is achieved when model formuliation, numerical methods, programming language and the computer are optimized as an integrated system. This means that one selects at first the simulation system and utilizes its advantages in the formulation of the mathematical model and in selecting the computing methods.

The integrated simulation facility of hardware and software must than operate until more advanced experimental results lead to advanced modeling methods and justify the acquisition of, and programming for, the next generation of simulation facility. It is more likely, however that the software of today's optimized integration facility will outperform current standard FORTRAN codes also on future computing platforms.

\section{SUMMARY}

It was demonstrated that engineering judgement in selecting and formulating models, in selecting established and documented numerical methods, and that working in a simulation environment, can significantly improve the quality of two-phase flow analyses, while reducing the time and cost that they require.

The paper explains the difference between computing and simulation and presents six principles of modeling and numerical methods, all of which were demonstrated to successfully reduce the computational effort. Three coding requirements are presented, and the important features of the simulation computer environment, primarily for intensive input and output processing. Lastly, it is shown why modeling and numerical methods need to be optimized with the computer architecture as a whole.

\section{REFERENCES}

[1] W. Wulff, H.S. Cheng, A.N. Mallen and U.S. Rohatgi, BWR stability analysis with the BNL Engineering Plant Analyzer, USNRC Report, Brookhaven National Laboratory, NUREG/CR-5816 (November 1991). 
[2] W. Wulff, H.S. Cheng, and A.N. Mallen, Causes of instability at LaSalle and consequences from postulated scrum thilure, Proc. OECD Intemutional Workshop on Boiling Water Reactor 5tability, Holtsville, NY, CSNI Report 178 (October 1990).

[3] W. Wulff, H.S. Cheng, and A.N. Mallen, Effect of power oscillations cin suppression pool heating during ATWS conditions, Proc. OECD International Workshop on Boiling Water Reactor Stability, Holtsville, NT, CSNI Report 178 (October 1990).

[4] W. Wulff, H.S. Cheng, S.V. Lekech and A.N. Mall ., The BWR Plant Analyzer, USNRC Report, Brookhaven National Loboratory, NUREG/CR-3943 (August 1984).

[5] G. Jhusem, S. Rouhuni and R. Schumway, A parametric study of variablis, afferting BWR stability, Proc. OECD Intermationtul Workshop on Boiling Water Reactor Stability, Y'oltsville, NY, CSNI Report 178 (October 1990).

[6] J.H. MoFadden et al, RETRAN-02, A program for transient thermal-hydraulic analysis of complex fluid flow systems, Electric Power Research Institute Repout NP-1850 (1981) ElPRI Palo Aito, CA.

[7] M.P. Paulsen and E.D. Hughes, Nonequilibrium model for operational trunsient analysis, Nuclear Technology, Vol. 61, No. 2 (1983).

[8] F.D. Hughes and K.R. Kutsma, Numevical methods improvernents for RETRAN, Nuclear Technology, Vol. 61, No. 2 (1983) 167.

[9] V.H. Ransom ef al, RELAP5/MOD2 code manual, USNRC Report NURE.j/CR-4312, Idaho National Engineering Laboratory, EG\&G Idaho, Inc., Idaho Falls, ID (1985).

[10] R.A. Dimenna et al, RELAP\%/MOD.2 models and correlations, USNRC, Report NUREG/CR-5194, Idaho National Engineering Laboratory, EG\&G Idaho Inc., Idaho Falls, ID (1985).

[11] J.A. Trapp and R.A. Riernke, Completion of nearly implicit hydrodynamic numerical scheme for RELAPS/MOD2, Intemal Technical Report SE-RSTD-85-006, Idaho Naticinal Engineering Laboratory, EG\&O Idaho Inc., Idaho Falls, ID (1985).

[12] TRAC-PF 1: an advanced best-estimate computer code for pressurized waller reastor analysis, USNRC Report NUREG/CR-3567, Los Alamos National Laboratory, Los Alamcis, NM (1984).

[13] D.R. Liles et al, TRAC-PF1 correlations and models, USNRC Repont NUREG/CR-5096, Los Alamos National Laboratory, Los Alamos, NM (1988).

[14] L. Mainstream, EKS EASY5 dynamic analysis system, Users guide, Boeing Computer Services Company, Ist Edition (March 1980).

[15] Mitchcil and Gauthier, Assoc. Inc., Advanced Continuous System Simulation Language (ACSL) - User Guide and Reference Manual, 3rd Ed. (1981).

[16] Applied Dynamics International (ADI), ADSIM Reference Manual, Version 7.1, (May 1990); ADI, Ann Arbor, Ml.

[17] E.J. Fadden, L.H. Michsels and A. Haraldsdottir, The Agplied Dynaınicsi Intenuational SYSTEM 100: an integrated continuous system simulation environment, Proc. Microprocesserr and Array Processor Confentuce, Tumpa, FL (1989). 
[18] W. Wulff, Major systems codes, carabilities and limitations, Proc. Simulation Methods for Nuclear Power Systems, Tuscon, AZ, US Nuclear Regulatory Commission and Electric Power Research Institute, EPRI WS-81 (May 1981).

[19] M. Ishii, Thermo-fluid dynamic theory of two-phase flow, Collection de 1\%. Directron des Eivdes ef Recherches d'Electricté de France, Eyrolles, Paris (1975) p. 189.

[20] M. Ishii, Two-fluid model for two-phase flow, in: Multiphase Science and Technology, Vol. V, od. G.F. Hewitt, J.M. Delhaye and N. Zuber (Hemisphere Publishing Corporation, New York, Washington, Philadelphia, London, 1990) pp. 1-63.

[21] N. Zuber and A.J. Findlay, Average volesmetric concentrations in two-phase flow systems, Journal of Heat Transfer, 87 (1965) 453.

[22] M. Ishii, One-dimensional drift-flux model and constitutive equations for relative motion between phases in various two-phase flow regimes, Argonne National Laboratory Report ANL-77-47 (October 1977).

[23] J. A. Boure, Wave phenonene and one-dimesinosil two-phase flow models, in: Multiphase Science and Technology, Prts 1, 2 and 3, Vol. VII, ed. G.F. Hewitt, J.M. Delhaye and N. Zuber (Hemisphere Publishing Corporation, New York, Washington, Philadelphia, London, 1991).

[24] J.E. Meyer and R.P. Rose, Application of a mementum integral model to the study of parallel channel boiling flow oscillations, Trans. ASME, J. Heat Transfer 85 (1963) 1.9.

[25] W. Wulff, Lumped parameter models for transient conduction in nuclear reactor components, USNRC Report NUREG/CR-1540, BNL-NUREG-35944, Brookhaven National Laboratory, Upton, NY 11973.

[26] W. Wulff, M.S. Cheng and A.N. Mallen, Analytical modeling lechriques for efficient heat transfer simulation in nuclear power plant transients, ANS Thermal Hydraulics Proc. 1985 National Heat Transfer Conference, Denver CO, (Aug. 1985), BNL-NUREG-35944, Brokhaven National Laboratory, Upton, NY 11973.

[27] W. Wulff, Computation:I Methods for Multiphase. Flow, in: Multiphase Science and Technology, Vol. V, ed. G.F. Hewitt, J.M. Delhaye and N. Zuber (Hemisphere Publishing Corporation, New York, Washington, Philadelphia, London, 1990) pp. 85-238 (see tuble 8.1 on p.155).

[28] J.L. Bryant aad N.P. Wilburn, Handbook of softiware quality assurance techniques applicable to the nuclear industry, Pacific Northwest Laboratory Report, NUREG/CR-4640 (August 1987).

\section{DISCILAIMER}

\footnotetext{
This report was prepared as an account of work spunsorod by an agency of the Unitod States Government. Neither the United States Government nor any agency thereof, not any of their employnes, makes any warranty, express or impliod, or assumes any legal liatrility or respontion bility for the accuracy, completeness, or usefulness of any information, apparatus, product, or process disclosed, or represents that its use would not infringe privately owned rights. Refer. ence bercin to any specific commercial product, process, or service by trade name, trademark. munufacturer, or otherwise does not necessarily constifute or imply its endorsement, reasmmeadation, of favoring by the United States Government or any agency thereof. The views and opinions of authors expressed herein do solt necessarily state of reflect thowe of the United States Goverwameal or any agency thereof.
} 

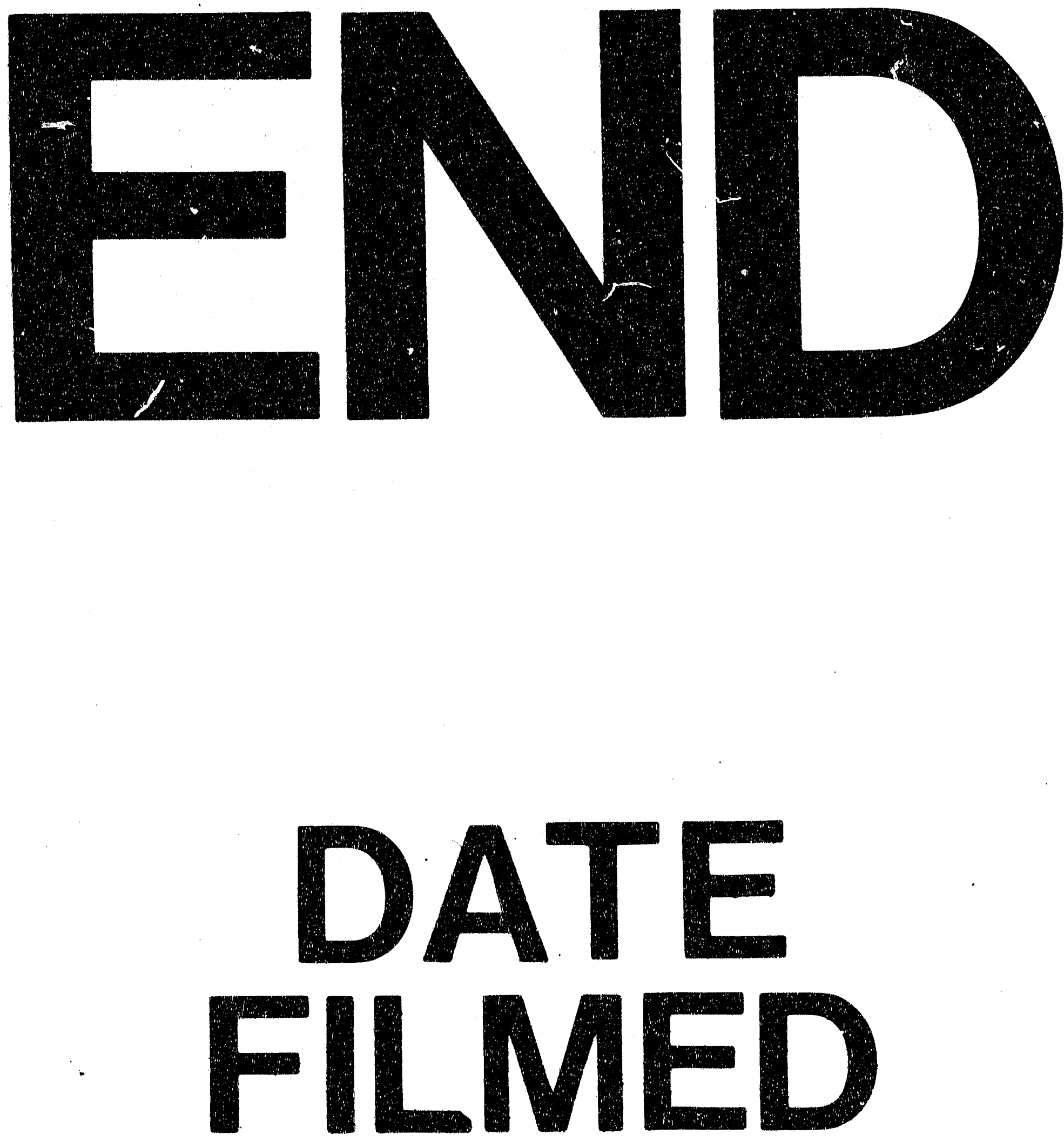

妻




